\title{
Validación del Cuestionario Breve de Confianza Situacional en muestra clínica con drogodependencia
}

\author{
Angel López Ortiz@', Marisela Gutiérrez Vega(D) \\ I Universidad Autónoma de Ciudad Juárez (UACJ), Chihuahua, México
}

\section{RESUMEN}

Introducción: numerosos estudios han concluido que la autoeficacia es una variable esencial dentro del área de drogadicciones y clave para el mantenimiento de la abstinencia y prevención de recaídas. El Cuestionario de confianza situacional, elaborado por Annis y colaboradores (1987), es uno de los instrumentos más utilizados para medir la autoeficacia y ha tenido diversas modificaciones. El Cuestionario Breve de Confianza Situacional (CBCS), en su versión más corta cuenta con sólo ocho ítems. Objetivo: obtener la validez factorial y fiabilidad del CBCS con muestra clínica en modalidad residencial. Método: el estudio contó con una muestra de 41 pacientes voluntarios, adolescentes y adultos, internos en un centro de rehabilitación en modalidad residencial. La evaluación cumplió con las normas para el desarrollo y revisión de estudios instrumentales. Resultados: al realizar el análisis factorial exploratorio se quedaron los ocho reactivos originales, compuestos en un sólo factor que explica $71.39 \%$ de la varianza, una carga factorial entre los rangos de .76 y .91 y con un índice de consistencia interna de $a=.941$. Discusión y conclusiones: el Cuestionario Breve de Confianza Situacional presenta una consistencia interna con alta fiabilidad y aceptables cargas factoriales, en similitud con lo encontrado en otros estudios realizados. Por lo que se sugiere su utilización en México como herramienta de evaluación en un ambiente clínico, con pacientes que sean internos en un centro de rehabilitación. Es recomendable realizar replicaciones con muestras más representativas y seleccionada aleatoriamente. Además, se sugiere incluir el análisis factorial confirmatorio para obtener mayor certeza en los resultados.

Palabras clave: validación, CBCS, consumo de drogas, tratamiento residencial, autoeficacia.

\begin{abstract}
Introduction: many studies have concluded that self-efficacy is an essential variable within the area of drug addictions and a key to maintaining abstinence and preventing relapses. The Situational Confidence Questionnaire by Annis et al. (1987), is one of the most used instruments to measure self-efficacy and has had various modifications. The Brief Situational Confidence Questionnaire (CBCS), is the shortest version with only 8 items. Objective: to obtain the factor validity and reliability of the CBCS with a clinical sample in residential modality. Method: the study was carried out with a sample of 41 volunteers patients, adolescents, and adults interned in a residential rehabilitation center. The evaluation met the standards for the development and review of instrumental studies. Results: after carrying out the exploratory factor analysis, the eight original items were left within a single factor which explains $71.39 \%$ of the variance, with a factor load between the ranges of .76 and .91, and with an internal consistency of .941. Discussion and conclusions: the Brief Situational Confidence Questionnaire presents an internal consistency with high reliability and acceptable factor loads, such as was found in other studies. Therefore, its use in Mexico is suggested as an evaluation tool in a clinical setting, with patients who are interned in a rehabilitation center. It is recommended to carry out replications with more representative and randomly selected samples. Furthermore, it is suggested to include confirmatory factor analysis to obtain greater certainty in the results.
\end{abstract}

Keywords: validation, BSCQ, residential treatment, self-efficacy, relapse prevention.

\section{Autor de correspondencia:}

Angel López Ortiz. Maestría en Psicología. Universidad Autónoma de Ciudad Juárez.

Teléfono celular: 656148 4418. Correo electrónico: angel.lopez.psy@gmail.com

Recibido: 16 de mayo de 2020

Aceptado: 28 de julio de 2020

doi: 10.28931/riiad.2020.2.02 


\section{INTRODUCCIÓN}

Aproximadamente, 5.6 por ciento de la población mundial ha consumido sustancias psicoactivas en el último año, de quienes al menos 11 por ciento presentan dependencia y requieren de un tratamiento (UNODC, 2018). En México, 8 de cada 100 consumidores de drogas acuden a un tratamiento de rehabilitación y solamente una cuarta parte de ellos lo finaliza (ENCODAT, 2017).

La recaída es el acto de volver a consumir de manera excesiva después de haber mantenido un periodo de abstinencia, y es una variable que representa un reto para los tratamientos de rehabilitación (Witkiewitz \& Marlatt, 2004). Su tasa de prevalencia es semejante a la de enfermedades crónicas, como la diabetes, el asma y la hipertensión. Usualmente, entre 40 y 60 por ciento de los pacientes con problemas de drogadicción recaen durante el primer año después del tratamiento (NIDA, 2010), aunque hay mayor probabilidad de presentar una recaída dentro de los primeros 90 días de abstinencia (Brooks \& McHenry, 2015).

Se ha concluido que para evitar recaídas se deben identificar situaciones de alto riesgo en donde se suele consumir, desarrollar habilidades de afrontamiento y aumentar la autoeficacia (Barragán Torres et al., 2014). Esta última variable se puede considerar como la percepción de la capacidad para conseguir un objetivo (Borland, 2014) y se ha concluido que los individuos que tienen bajos niveles de autoeficacia usualmente presentan mayor probabilidad de involucrarse en conductas de riesgo (Palacios Delgado, 2015). En el área de adicciones se ha encontrado que cada vez que un individuo logra afrontar situaciones de riesgo que se le presentan, y mantiene su abstinencia de no consumo, su nivel de autoeficacia aumenta, incrementando las posibilidades de no recaer ante esa situación (Witkiewitz \& Marlatt, 2004). Diversos autores han observado que los individuos que presentan niveles bajos de autoeficacia suelen evadir las situaciones de riesgo que se les presentan, así como experimentar mayores niveles de consumo de alcohol y otras sustancias psicoactivas (López-Torrecillas et al., 2015). Según la revisión teórica de Castro (2012), se ha concluido que los individuos con alta autoeficacia son menos propensos a tener una recaída, logrando mantener su abstinencia por al menos seis meses después del tratamiento (Castro Deza, 2012).

Marlatt y colaboradores encontraron que existen cinco categorías de autoeficacia que se relacionan con las conductas de consumo de drogas: a) resistencia; b) reducción de daños; c) acción; d) afrontamiento; e) recuperación (Marlatt et al., 1995). Por otro lado, el cambio favorable en la autoeficacia puede darse a través de cuatro diferentes formas: a) la experiencia de haber lo- grado una meta y objetivo; b) a través de la experiencia de observar y creer que otras personas han afrontado exitosamente situaciones similares a la propia; c) recibir motivación por medio del lenguaje verbal de otras personas; d) experimentar un equilibrio emocional, permitiendo tener un criterio objetivo acerca de las propias capacidades (López-Torrecillas et al. 2002).

Uno de los instrumentos más utilizados para medir la autoeficacia es el Cuestionario de Confianza Situacional (CCS), elaborado por Annis y Graham en 1987 (Barragán Torres et al., 2005). La versión original estuvo compuesta por 100 ítems, sin embargo, han surgido versiones más breves. Una de ella estuvo compuesta por 50 ítems y fue aplicada inicialmente a una muestra de 713 pacientes que estaban en tratamiento por consumo de sustancias. Con base en los resultados del análisis factorial exploratorio, así como del confirmatorio, se concluyó que estaba compuesta por ocho factores, asignándoles una subescala a cada uno, con una buena fiabilidad, con alfas de Cronbach entre .79 y .95 (Sklar et al., 1997). Otros estudios han concluido que este instrumento de 50 ítems cuenta con $a=.98$, sugiriendo que se puede reducir a ocho ítems o inclusive a tres, y se obtendrían los mismos resultados (Chicharro Romero et al., 2007). Posteriormente, se obtuvo una versión de ocho ítems; el Cuestionario Breve de Confianza Situacional (CBCS), de un sólo factor y consistencia interna similar a la versión original (Breslin et al., 2000).

Además de adultos, el CBCS ha sido validado en menores de edad y se ha obtenido una consistencia interna de $a=.84$ y .86 , respectivamente. Con base en la regresión binomial negativa, se observó que los puntajes del instrumento se relacionan con el consumo de sustancias. Sus puntajes mostraron suficiente estabilidad, consistencia interna y validez (Delaney et al., 2020). Adicionalmente, se ha validado en población con enfermedades mentales severas (O'Hare \& Shen, 2013).

En el caso de México, la versión breve de ocho ítems fue adaptada en 1977 por Echeverría, con una consistencia interna de .97 de alfa de Cronbach (Echeverría \& Ayala, 1977). Posteriormente, en un estudio realizado con 1,175 universitarios consumidores de alcohol, se obtuvo una versión de cuatro ítems y escala tipo Likert, presentando una consistencia interna de .78 y una sola dimensión (Salazar Garza et al., 2019).

Debido a que la autoeficacia es la variable más relevante y efectiva para el control de conductas adictivas, (Hinojosa-García, et al., 2012) es de especial relevancia contar con instrumentos válidos y fiables que permitan su medición en diferentes contextos, por lo que el objetivo del presente estudio es obtener la validez factorial y fiabilidad del CBCS con muestra clínica en modalidad residencial. 


\section{MÉTODO}

\section{Participantes}

Considerando los procedimientos factoriales que se realizaron con el instrumento para analizar sus propiedades de fiabilidad y validez, se contó con un mínimo de cinco participantes por ítem (Nunnally \& Bernstein, 1995), quedando conformada la muestra por 41 pacientes de un centro de rehabilitación, ubicado en Ciudad Juárez, Chihuahua, con un tipo de muestreo por conveniencia. La muestra estuvo conformada por 38 hombres y tres mujeres; de los cuales 51.2 por ciento eran menores de edad, con un promedio de 16.4 años, y 48.8 por ciento eran adultos, con un promedio de 37.1 años. Todos los participantes eran poliusuarios, sin embargo, el cannabis fue la droga de impacto de 45 por ciento de los participantes, la cocaína fue la droga de impacto para 33 por ciento de ellos, y para el 22 por ciento restante, fue el cristal.

\section{Instrumentos}

El CBCS es una versión breve del CCS elaborado por Annis y Graham en 1987. Originalmente estuvo compuesto por 100 ítems, y ambas versiones fueron diseñadas para evaluar la autoeficacia en ocho situaciones que los clientes reportaron como desencadenantes de recaídas: a) emociones agradables; b) malestar físico; c) probar autocontrol; d) necesidad de consumo; e) momentos agradables con otros; f) conflicto con otros; $g$ ) emociones desagradables; h) presión social. Tanto en el CCS como en el CBCS se han observado niveles de consistencia interna similares (Breslin et al., 2000).

\section{Procedimientos}

Se acudió a un centro de rehabilitación reconocido por la Comisión Nacional contra las Adicciones (CONADIC), en donde se explicó al personal directivo el objetivo del estudio y cuestiones sobre la confidencialidad de los resultados. Después de obtener la autorización institucional, se contactó a los tutores legales de cada participante menor de edad, quienes autorizaron su participación. Posteriormente, se realizó una sesión informativa con los participantes, donde se explicó nuevamente el objetivo del estudio, confidencialidad y se les hizo saber que en cualquier momento podrían interrumpir su participación, en caso de así desearlo. Finalmente, se les entregó un consentimiento informado, el cual fue firmado por todos los participantes.

La aplicación del instrumento fue de manera grupal, separando a los participantes en cuatro grupos, con el fin de facilitar la explicación de instrucciones y dudas referente al llenado del instrumento. Con base en la guía de usuario que publicaron Annis y Graham en 1998, en cada ítem se le pidió al participante que evaluara una situación de alto riesgo con relación a su confianza o seguridad, para resistirse a consumir drogas en dicha situación, bajo una escala de 0 por ciento; "totalmente inseguro", a 100 por ciento; "Totalmente seguro" (Hayaki et al., 2011).

Se utilizó el programa estadístico IBM-SPSS 25 para obtener el índice de validación de confiabilidad con consistencia interna y se realizó un análisis de fiabilidad utilizando el modelo de alfa y la validez de constructo, a través de un análisis factorial exploratorio (AFE), mediante el método de mínimos cuadrados no ponderados y analizando el matriz de correlaciones, basado en autovalores mayores que 1. Con referente a la rotación, se usó el Oblimin directo por ser la rotación oblicua más utilizada (Lloret-Segura et al., 2014). Adicionalmente, se consideraron las pruebas de KMO y Barlett.

\section{RESULTADOS}

Los resultados del AFE indican que la medida Kaise-Meyer-Olkin de adecuación de muestreo fue de .890 , lo que corrobora que las matrices del modelo fueron factorizables, considerando que valores entre 0.5 y 1.0 indican un análisis factorial apropiado (Cerny \& Kaiser, 1977). Por otro lado, la prueba de esfericidad de Bartlett fue de $260.451(p=<.001)$, lo que provee evidencia adicional de que la matriz de correlación fue adecuada para el análisis factorial. Al llevar a cabo el AFE no se eliminó ningún ítem, es decir quedaron los ocho reactivos con comunalidades de extracción entre los rangos de .580 a .836, lo que sugiere una relación entre los reactivos. Se compone de un factor que explica el 71.395 por ciento de la varianza con un alfa de Cronbach total de .941. Se utilizó el método de extracción de cuadrados mínimos no ponderados, obteniendo una carga factorial de cada ítem entre rangos de .762 y .914.

Tabla 1

Matriz factorial

\begin{tabular}{lc}
\hline Autoconfianza de no consumir ante: & Factor 1 \\
\hline Emociones desagradables & .783 \\
Malestar físico & .851 \\
Emociones agradables & .802 \\
Probar el autocontrol & .762 \\
Necesidad de consumo & .810 \\
Conflicto con otros & .771 \\
Presión social & .864 \\
Momentos agradables con otros & .914 \\
\hline
\end{tabular}




\section{DISCUSIÓN Y CONCLUSIÓN}

La principal contribución de este estudio fue obtener un instrumento válido para pacientes internos en un centro de rehabilitación de drogas bajo modalidad residencial, integrado por una sola dimensión con ocho reactivos, lo que concuerda con estudios previamente realizados (Breslin et al., 2000; Cerny \& Kaiser, 1977; Sklar et al., 1997). A pesar de que la versión breve del cuestionario ha sido utilizada en diversas intervenciones, e inclusive en guías como el "Manual de prevención de recaídas" emitido por la Secretaría de Salud del gobierno federal mexicano (Barragán Torres et al., 2014), se cuenta con pocos datos sobre el índice de consistencia interna de esta versión del instrumento (Salazar Garza et al., 2019).

Una posible desventaja de este estudio es que el CBCS se aplicó en un rango de edad amplio; sin embargo, los resultados sugieren una consistencia interna aceptable para su uso en adolescentes y adultos. En otras investigaciones se ha utilizado en adolescentes con edad promedio entre los 15 y los 16 años (Blanco Álvarez \& Jiménez Morales, 2015; Martínez Martínez et al., 2008; 2010; Rodríguez Durán et al., 2017), o en población adulta (Chicharro Romero et al., 2007), con edad promedio de 35 años (Barragán Torres et al., 2005), de 39.8 años (Breslin et al., 2000), o edad promedio de 50 años (Sobell et al., 1996).

Asimismo, es necesario realizar estudios similares en donde se contemple una muestra más amplia, seleccionados aleatoriamente, y además del análisis factorial exploratorio se realice el análisis factorial confirmatorio. Así como la realización de estudios que contemplen otras variables como situaciones de riesgo o estrategias de afrontamiento, con el fin de obtener datos que sirvan para la realización de programas de prevención.

\section{DECLARACIONES DE LOS AUTORES}

No existe ningún tipo de conflicto de interés.

\section{FUENTES DE FINANCIAMIENTO}

No se obtuvo ningún financiamiento para realizar el presente estudio.

\section{REFERENCIAS}

Barragán Torres, L., Flores Mares, M., Ramírez García, A. N. \& Ramírez Mendoza, C. (2014). Manual del programa de prevención de recaídas. Secretaría de salud.

Barragán Torres, L., González Vázquez, J., Medina-Mora, M. E. \& Ayala Velázquez, H. (2005). Adaptación de un modelo de intervención cognoscitivo-conductual para usuarios dependientes de alcohol y otras drogas a población mexicana: un estudio piloto. Salud Mental, 28(1), 61-71.

Blanco Álvarez, T. \& Jiménez Morales, S. (2015). Factores de riesgo que influyen en la recaída de consumo de drogas lícitas e ilícitas en adolescentes atendidos en el Instituto sobre Alcoholismo y Farmacodependencia. Revista Costarricense de Psicología, 34(2), 147-157.

Borland, R. (2014). Conceptual influences on change. En R. Borland (Ed.), Understanding hard to maintain behaviour change, a dual process approach (pp. 117-139). Wiley Blackwell.

Breslin, F. C., Sobell, L. C., Sobell, M. B. \& Agrawal, S. (2000). A comparison of a brief and long version of the Situational Confidence Questionnaire. Behavior Research and Therapy, 38(12), 1211 1220. doi: 10.1016/S0005-7967(99)00152-7

Brooks, F. \& McHenry, B. (2015). A contemporary approach to substance use disorders and addiction counseling. American Counseling Association.

Castro Deza, L. (2012). Autoestima y autoeficacia en pacientes adictos en el centro de rehabilitación de ñaña. Revista de psiquiatría y salud mental "Hermilio Valdizán", 13(2), 3-21.

Cerny, B. A. \& Kaiser, H. F. (1977). A study of a measure of sampling adequacy for factor-analytic correlation matrices. Multivariate Behavioral Research, 12(1), 43-47. doi: 10.1207/s15327906mbr1201_3

Chicharro Romero, J., Pedrero Pérez, E. J. \& Pérez López, M. (2007). Autoeficacia para resistirse al consumo de sustancias como predictora de resultados de tratamiento y su relación con variables de personalidad: estudio de una muestra de adictos con el DTCQ, el VIP y el MCMI-II. Adicciones, 19(2), 140-151. doi: 10.20882/adicciones.312

Delaney, D. J., Bernstein, M. H., Harlow, L. L, Farrow, M., Martin, R. A. \& Stein, L. A. R. (2020). The brief situational confidence questionnaire for alcohol: A psychometric assessment with incarcerated youth. Psychological Assessment, 32(3), 254-264. doi: 10.1037/ pas0000780

Echeverría, L., \& Ayala, H. (1977). Cuestionario de confianza situacional: traducción y adaptación. Universidad Nacional Autónoma de México.

Instituto Nacional de Psiquiatría Ramón de la Fuente Muñiz; Instituto Nacional de Salud Pública; Comisión Nacional contra las Adicciones, Secretaría de Salud (2017) Encuesta Nacional de Consumo de Drogas, Alcohol y Tabaco 2016-2017: Reporte de Drogas. INPRFM

Hayaki, J., Herman, D. S., Hagerty, C. E., de Dios, M. A., Anderson, B. J. \& Stein, M. D. (2011). Expectancies and self-efficacy mediate the effects of impulsivity on marijuana use outcomes: An application of the acquired preparedness model. Addictive Behaviors, 36, 389-396. doi: 10.1016/j.addbeh.2010.12.018

Hinojosa-García, L., Alonso-Castillo, M. M. \& Castillo-Muraira, Y. (2012). Autoeficacia percibida y consumo de alcohol en trabajadores de la salud. Revista de enfermería del Instituto Mexicano del Seguro Social, 20(1), 19-25.

Lloret-Segura, S., Ferreres-Traver, A., Hernández-Baeza, A. \& Tomás-Marco, I. (2014). Análisis factorial exploratorio de los ítems: 
una guía práctica, revisada y actualizada. Anales de Psicología, 30(3), 1151-1169.

López-Torrecillas, F., del Mar Salvador, A. V. \& Cobo, P. (2002). Autoeficacia y consumo de drogas: una revisión. Psicopatología clínica, legal y forense, 2(1), 33-51.

López-Torrecillas, F., Torres Cobo, M. A., Delgado, P. \& Ramírez Ucles, I. (2015). Predictive capacity of self-efficacy in drug dependence and substance abuse treatment. Journal of psychology and clinical psychiatry, 2(3), 1-7. doi: 10.15406 /jpcpy.2015.02.00073

Marlatt, A. A., Baer, J. S. \& Quigley, A. A. (1995). Self-efficacy and addictive behavior. En A. Bandura (Ed.), Self-efficacy in changing societies (pp. 289-316). Cambridge University Press.

Martínez Martínez, K. I., Pedroza Cabrera, F. J., Salazar Garza, M. L., \& Vacio Muro, M. (2010). Evaluación experimental de dos intervenciones breves para la reducción del consumo del alcohol de adolescentes. Revista mexicana de análisis de la conducta, 36(3), 35-53. doi: 10.5514/rmac.v36.i3.03

Martínez Martínez, K. I., Salazar Garza, M. L., Pedroza Cabrera, F. J., Ruíz Torres, G. M., \& Ayala Velázquez, H. E. (2008). Resultados preliminares del programa de intervención breve para adolescentes que inician el consumo de alcohol y otras drogas. Salud Mental, 31(2), 119-127.

National Institute on Drug Abuse (2010). Principios de tratamientos para la drogadicción: Una guía basada en las investigaciones. Recuperado de https://www.drugabuse.gov/es/publicaciones/principios-de-tratamientos-para-la-drogadiccion/enfoques-con-base-cientifica-para-los-tratamientos-de-la-dro-10

Nunnally, J. \& Bernstein, I. (1995). Teoría psicométrica. Mc Graw Hill

O’Hare, T., \& Shen, C. (2013). Abstinence self-efficay in people with severe mental illness. Journal of Substance Abuse Treatment, 45, 76-82. doi: 10.1016/j.jsat.2012.12.014
Palacios Delgado, J. R. (2015). Estimación psicométrica de la escala de autoeficacia ante conductas de riesgo para adolescentes en México. Psychosocial Intervention, 24(1), 1-7. doi: 10.1016/j. psi.2014.11.004

Rodríguez Durán, C. A., Echeverría San Vicente, L., Martínez Martínez, K. I., \& Morales Chainé, S. (2017). Intervención breve para adolescentes que inician el consumo abusivo de alcohol y otras drogas: su eficacia en un escenario clínico. Revista Internacional de Investigación en Adicciones, 3(1), 16-27. doi: 10.28931/ riiad.2017.1.03

Salazar Garza, M. L., Vacio Muro, M. \& Macías Rodríguez, J. J. (2019). Adaptación y validación del Cuestionario breve de confianza situacional en universitarios mexicanos que consumen alcohol. Revista Internacional de Investigación en Adicciones, 5(2), 30-37. doi: 10.28931/riiad.2019.2.04

Sklar, S. M., Annis, H. M \& Turner, N. E. (1997). Development and validation of the drug-taking confidence questionnaire: A measure of coping self-efficacy. Addictive Behaviors, 22(5), 655,670. doi: 10.1016/s0306-4603(97)00006-3

Sobell, L. C., Cunningham, J. A., Sobell, M. B., Agrawal, S., Gavin, D. R., Leo, G.I. \& Singht, K. N. (1996). Fostering self-change among problem drinkers: a proactive community intervention. Addictive Behaviors, 21(6), 817-833. doi: 10.1016/03064603(96)00039-1

United Nations Office on Drugs and Crime (2018). Global overview of drug demand and supply. World Drug Report 2018.

Witkiewitz, K. \& Marlatt, G. A. (2004). Relapse prevention for alcohol and drug problems: That was Zen, This is Tao. American Psychologist, 59(4), 224-235. doi: 10.1037/0003-066X.59.4.224 